\title{
Reducing shoulder injuries among construction workers
}

\author{
Abdullatif Alwasel $^{1 *}$, Karim Elrayes ${ }^{2}$, Eihab Abdel-Rahman ${ }^{1}$, and Carl Haas ${ }^{3}$ \\ ${ }^{1}$ Systems Design Engineering, University of Waterloo, Canada \\ ${ }^{2}$ Electrical and Computer Engineering, University of Waterloo, Canada \\ ${ }^{3}$ Civil and Environmental Engineering, University of Waterloo, Canada \\ *Corresponding author (aalwasel@uwaterloo.ca)
}

\begin{abstract}
Purpose The U.S. Bureau of Labor1 2008 report stated that the number of workers aged 65 or older is expected to rise by $36.6 \%$ from 2006 to 2016 . This rise is expected to accompany a $6.9 \%$ decrease in the number of workers between ages 16 to 24 and a slight increase by $2.4 \%$ for workers ages 25 to 54 . This indicates that the industry will face an aging workforce problem that can affect capacity and productivity if workers' health is not properly monitored. An essential problem for the aging workforce is musculoskeletal disorders (MSDs), specifically work-related musculoskeletal disorders (WMSDs). In 2008, shoulder WMSDs constituted 6.9\% of all WMSDs in construction, and this percentage was substantially higher for crafts such as electricians, carpenters, and painters. The main risk factor for shoulder WMSDs is prolonged forceful overhead work. This paper is presents a method to monitor shoulder WMSDs risk factor development and to act as an alarm to prevent the disorder from happening. Method An exoskeleton is being developed that will be worn by a worker to wirelessly track upper-arm motion in 3 dimensions using an array of anisotropic magneto- resistive (AMR) sensors, deployed over workers' upper-arms. The sensory system will be controlled using a microcontroller with the ability to save data on an SD card for post-processing. Results \& Discussion Alwasel et al. measured the angle of the upper-arm to trunk using AMR-sensors in an unpublished thesis. The problem with the use of AMR was the assembly itself since the sensor was not held at the center of joint rotation. However the device presented here will place AMRsensors at the center of joint rotation by extending the joint axis of rotation outside the body. This will provide enough data to help assess whether a worker is at risk of developing shoulder injury. This technique will help reduce the number workers that leave the workforce because of injury, and help maintain a good working environment. This technique will also assist in developing and applying health and safety guidelines to overhead work. Thus, it may ultimately help extend the productive age of the construction workforce.
\end{abstract}

Keywords: gerontology, safety, robotics

\section{INTRODUCTION}

Recently the number of industry, equipment, and tools has increased significantly as a result more workers are joining the workforce everyday to fulfill the increasing demand. The increase in the workforce poses a threat on workers' health, world industry, and insurance companies. The U.S. Bureau of Labor $^{1} 2008$ reported that the number of workers, age 65 or older, is expected to rise in 2016 by $36.6 \%$ from what it was in 2006. This rise is expected to accompany a $6.9 \%$ decrease in the number of workers between ages 16-24 years, and an increase by $2.4 \%$ for workers between ages $25-54$ years. The rise indicates that the industry will face an aging workforce problem that can affect capacity and productivity if workers' health is not properly monitored. The report also shows that $29 \%$ of all injuries and illnesses, that required days out of work, were diagnosed as musculoskeletal disorders (MSDs). Out of all MSDs reported, 6.9\% affected workers' shoulders. This percentage significantly increases for crafts such as painters, carpenters, and electricians because these workers spend significant amount of time working above head level which is often reported as the leading risk factor for Shoulder injuries ${ }^{2,4-9}$. Many health and safety organizations ${ }^{5-7}$ attempted solving the uprising problem over the years to reduce the impact on health and industry by: publishing safety manuals and guidelines that explain how to perform ergonomically safe tasks, arranging for seminars and workshops to increase the workers' awareness, and suggesting the redesign of some workstations and tasks to be ergonomically safe for the worker. These attempts focused on decreasing the workers' exposure to MSDs risk factors by educating the individuals.

These guidelines, workshops, and seminars were proven theoretically to reduce the rate of injury among workers on the field. However, when deployed in the filed their effect was not seen. There is no drop in the percentage of incidents registered for MSDs. In contrary, the percentage is rising. 
Many reasons contributed in making these techniques not successful when deployed in the field, for example:

- Workplaces design complexity.

- Workers' level of education.

- Psychological barriers.

- The cost required for monitoring workers' behavior throughout the workday.

Therefore, the need for a monitoring method to track the MSDs risk factor and act accordingly to decrease the number of injuries in construction fields is essential. However, this solution has to be consistent with the pace of construction industry by:

- Being costly effective for the industry.

- Being deployable on the field.

- Causing no conflict with workers' performance.

- Causing no psychological consequences.

In this paper a new method to decrease the shoulder WMSDs is proposed. Since shoulder injury is a major type of WMSDs that affect workers during their work lifetime. This method is going to focus on decreasing the number of injuries affecting workers' shoulders. Monitoring the risk factors leading to the injury and indicating whether a worker is following the guidelines for ergonomically safe work. Reducing the number of injuries in the field is going to reduce the economic and health impact of the injury on the industry.

Tools vibration, repetitive movement, forceful work, and working with hands above head level were reported as risk factors causing shoulder WMSDs ${ }^{8}$. However, tools' vibrations along with repetitive movements were not highly associated with the injury. In contrast, working with hands above head level combined with forceful work was strongly associated with the shoulder WMSDs ${ }^{8}$.

Considering that tasks performed in a construction sites often involve engaging the worker with heavy tools/objects, it is fair to rate construction tasks as forceful tasks. Therefore, prolonged elevation of the arm above the head level is variable that leads to shoulder WMSD.

As a result, whenever a method proves its ability to monitor the time spent by a worker in awkward posture (working above head level) the number of injuries can be controlled. Managing the injury can be achieved by changing the type of tasks the worker is performing for a period of time, based on published data ${ }^{9}$, enough for the muscles to regain its original shape and condition.

\section{METHOD}

The objective of this paper is to provide a real-time tracking device that can be implemented in construction field. Accordingly, managing the MSDs risk fac- tors, and decreasing the number of incidents resulting from the prolonged forceful overhead work.

In an unpublished thesis by Alwasel $2011^{4}$, multiple sensors have been investigated for their feasibility to be used as the sensing element in tracking workers' posture in construction sites. Because of its durability, precision, simplicity, size, and cost, KMA200 Philips sensor is the most suitable sensor to be used in tracking workers' posture in construction sites ${ }^{4}$. The KMA200 advantages and disadvantages were compared to those of gyroscopes, accelerometers, magnetometers, and ultrasonic sensors ${ }^{4}$. Alwasel 2011 used the anisotropic magneto-resistive sensor (AMR) by placing the sensor in the human axilla and attaching the magnet source to the interior side of the upper arm. Author reported that this arrangement showed reliable qualitative results that can point out whether a worker's arm is higher or lower than a preset angle; in that case the limit was $90^{\circ}$ of arm flexion in sagittal plane. Figure 1 shows how the system performed when implemented on a single participant for several movements.

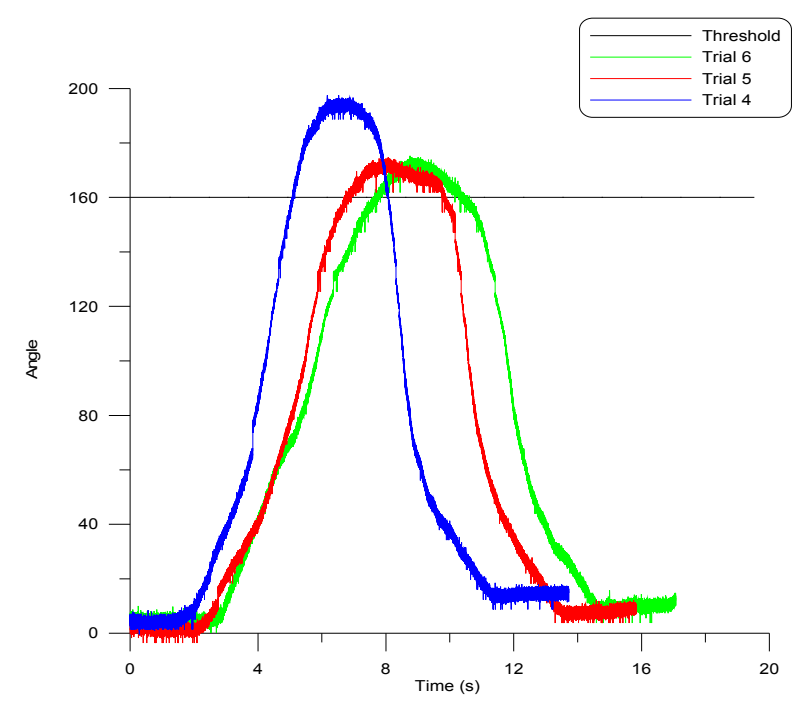

Fig.1. Three trials by a single participant aiming for higher $90^{\circ}$ of elevation recorded using Alwasel 2011 system ${ }^{4}$.

In figure 1 the participant was asked to wear the device and perform the same motion several times. In each motion the participant was asked to elevate his hands higher than a marker drawn on the wall corresponds to $90^{\circ}$ of upper arm to torso angle. The participant repeated the motion 9 times. The line shown at $160^{\circ}$ shown in figure 1 corresponds to $90^{\circ}$ degree of arm elevation. Because of the way that sensor was mounted with respect to the joint center, this system was not able to directly provide quantitative angles. It rather provided qualitative data showing whether the participant exceeded a certain angle $\left(90^{\circ}\right)$.

The problem of the previous system was that the AMR sensors have to be at center of joint rotation. 
This way the sensor would behave like it did when measuring a motor rotation with the sensor held at the center of rotation shown in figure 2 .

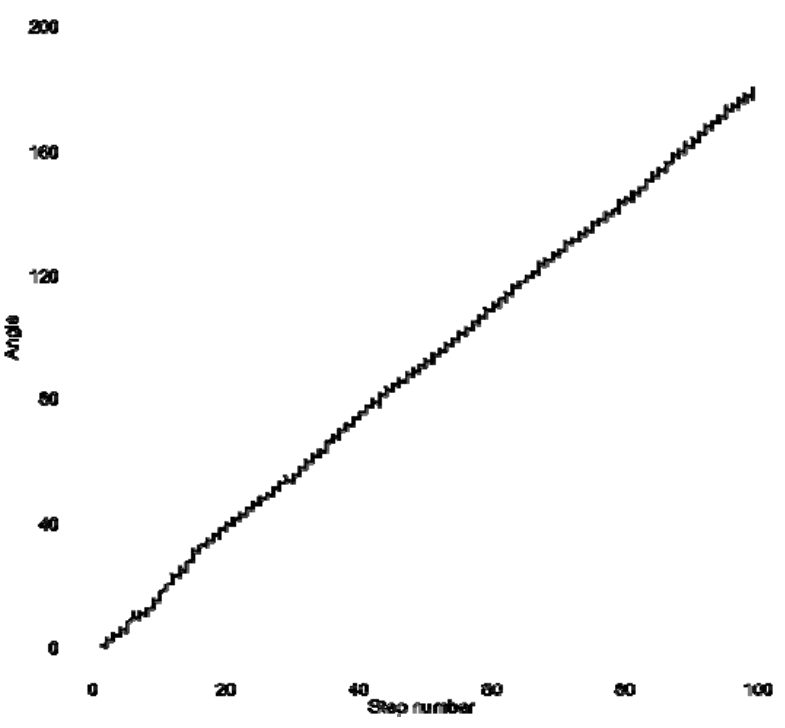

Fig.2. A $180^{\circ}$ motor rotation recorded by the KMA200 sensor where the sensor was held at the center of motor rotation ${ }^{4}$.

Producing such results needs either the sensor or the magnet to be invasive, which makes this solution not feasible. Therefore, this paper is suggesting the extraction of human joint centers outside the human body.

\section{D motion tracking exoskeleton}

This paper will explain the approach to extract the human joint center outside of the human body. This approach involves the design of exoskeleton that will ensure that the sensor is implemented in the center of the joint rotation.

Alwasel 2011 system was able to qualitatively measure the motion only in sagittal plane (2D), which lacks motions in frontal plane and motions that result from combination of movement in frontal and sagittal planes. In contrary, presented exoskeleton will be able to track the motion of the joint in 3D.

The exoskeleton is consisted of multiple linkages and hinges to extract the center of joint rotation outside the human body, thus placing the sensor noninvasively. For the shoulder rotation in sagittal plane (Flexion/Extension), the exoskeleton will have a linkage attached to the upper arm to move with its movement. The end of this linkage shown in figure 3 is a hinge-like joint where the magnetic source will rotate with the upper arm flexion. AMR sensor is held stationary at another linkage that extends from the shoulder area. This arrangement will act like the motor and sensor assembly shown in figure 4 .

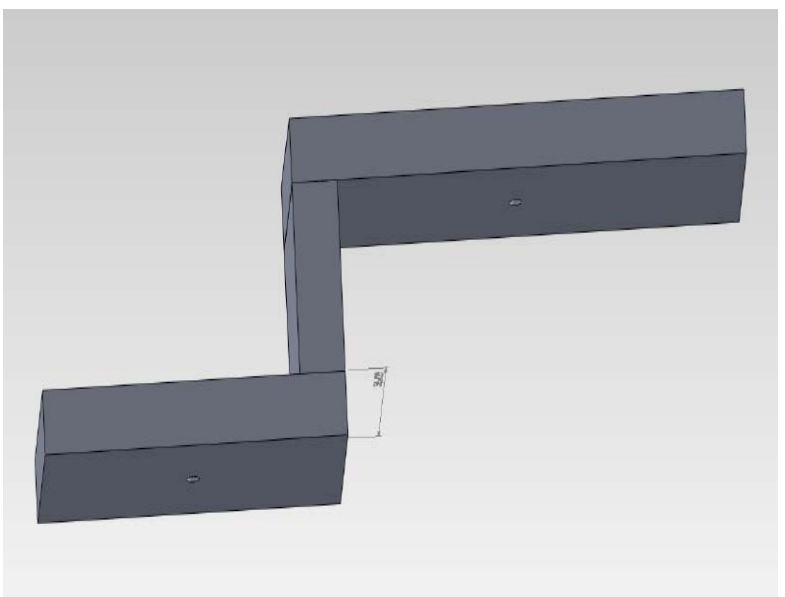

Fig.3. A hinge-like joint that will be placed at the end of upper arm linkage.

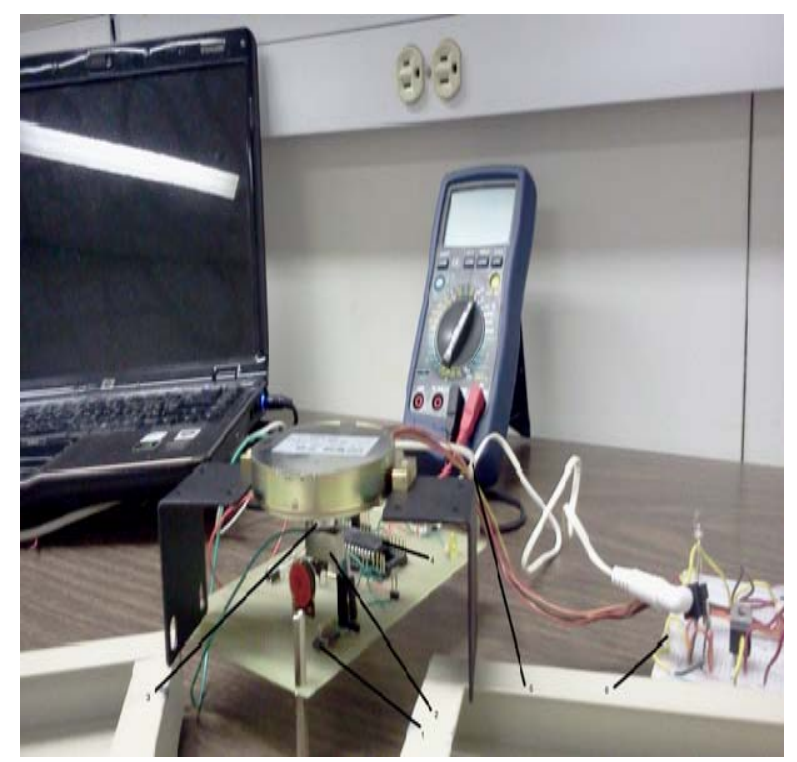

Fig.4. A single motor placed on top of the KMA200 sensor.

The other shoulder rotation (Abduction/Adduction) takes place in the frontal plane. This rotation will be detected using the same arrangement with different positions. There will be a linkage extending from the shoulder area to the upper arm, a sensor will be stationary and the magnet rotates with the upper arm rotation in frontal plane. Combining the two rotations, this exoskeleton will be able to mimic the results obtained from the motor movement except the exoskeleton will be three-dimensional. Figure 5 shows what the extraction of the joint center means. 


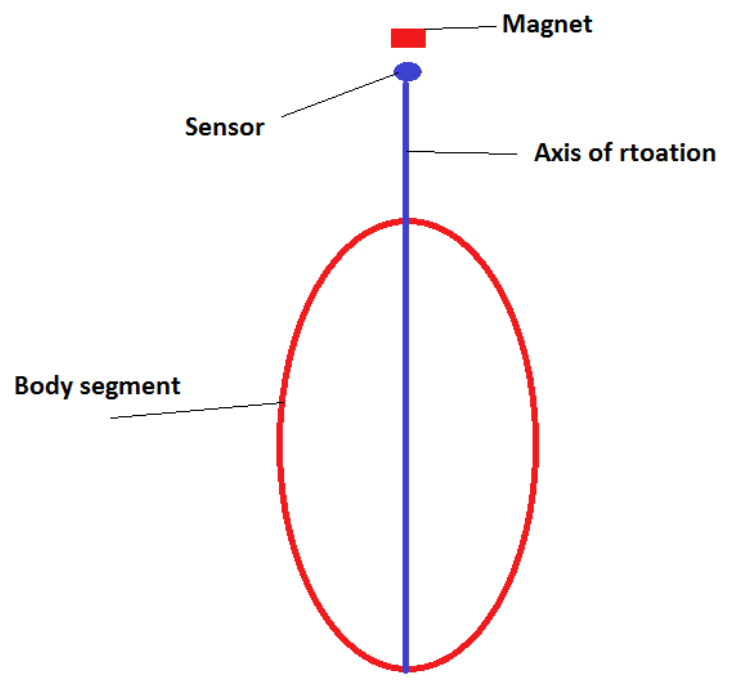

Fig.5. Extending the axis of rotation outside the body using external linkage (blue) to be able to mount the senor and magnet in the joint center noninvasively.

\section{Instrumentation}

Two programmable KMA200 angle sensors will be used as the sensing element in the exoskeleton. It requires an electrical control unit (ECU) to control the data flow, a memory to store the data, a power supply, a magnet source to generate the magnetic field lines, and an external case to protect the system and to carry it around. Two-500 gauss off-theshelf magnets will be used to create the saturation level needed to saturate the internal magnetization of the sensing element ${ }^{3}$.

The ECU uses an 80 pins microcontroller -MCU(PIC18F87J50) that has the ability to communicate to/and from the sensing element in digital and analog form if needed. Further, the MCU includes a buffer stack that is able to save data temporarily. The power requirement for the $\mathrm{MCU}$ is fulfilled using a $9 \mathrm{~V}$ battery along with a voltage regulator to decrease the voltage to the $5 \mathrm{~V}$ required by the $\mathrm{MCU}$.

Once sensors and magnets are in their respective positions they describe the relation between a moving frame and a stationary reference frame. As the upper arm moves (rotates) from 0 to $180^{\circ}$ the magnetic field lines rotate with the same angle resulting in change in resistance of the permalloy which follows equation 1.

$$
\mathrm{R}=\mathrm{R}_{\mathrm{o}}+\Delta \mathrm{R}_{\mathrm{o}}{ }^{*} \cos ^{2} \alpha
$$

where $R_{0}$ and $\Delta R_{0}$ are the base resistance and the coefficient of resistance as a function of flux, respectively, and $\alpha$ is the angle between the magnetic flux lines and the current ${ }^{10}$.

\section{Data acquisition}

The change in orientation of the applied magnetic flux-lines result in change in the internal resistances of the permalloy of the KMA200. This change in resistance is what this sensor use to calculate the angle according to equation (1). The KMA200 has the option of outputting the angle in digital or analog form.

In this exoskeleton the angle will be analog, this signal would be sent to a 16-bit analog to digital (ADC) converter for sampling at 100 sample/s. The output of the ADC would be sent to an SD card for storage.

\section{Discussion}

The need for a wireless, reliable, cheap, and easy to use motion tracking system is growing not only for shoulder injuries prevention only but also for many applications for example rehabilitation and sports training. This exoskeleton configuration can be applied to other joints in the human body. With minor changes in the linkage positions, this exoskeleton can measure the flexion/extension angle of elbow, knee, or foot.

There many options in the market currently that can track human motion wirelessly. Most of them use the inertial momentum unit technology (IMU). This technology uses assembly of gyroscopes, accelerometers, and magnetometers to track every segment in real time for every axis to get a three dimensional angle ${ }^{10}$.

The problem with systems that uses the IMU technology such as Xsene MVN ${ }^{10,11}$ is the complexity of mathematical operation that they use to extract the required angle requires a powerful control unit. Usually such systems uses mathematical filters such as Kalman filter to compensate the drift of the gyroscope ${ }^{12,13}$. They use a model that predicts the rotation based on tri-axial accelerometers and then refresh the gyroscope to cancel the drift. This requires a portable control unit that makes the system bulky. Further, the cost of having an IMU system is not feasible if, for example, it would be used to track motion of multiple workers in a construction site. Also the portability of such systems is limited to a maximum of 150 meters from the control unit.

However, the 3D motion tracking exoskeleton uses a single sensor and does not need any complicate mathematical operations to fuse signals or to correct drift. Also it does not require a powerful control unit as it can be managed with a single microcontroller. The cost of the 3D motion tracking system is less than the IMU systems, as it requires fewer components/sensors and controllers. Therefore, the presented exoskeleton is a good candidate to be used in tracking human body segments wirelessly.

The exoskeleton idea is to extract the joint center of rotation outside the human body. Accordingly magnet and sensor could be implemented in their respective positions. As a result, the exoskeleton will produce quantitative angles that can be used in science for 
research where the need for a wireless motion tracking is highly needed. Scientists currently use the motion capturing techniques that uses markers to identify many points over the human body. 3D coordinates for each marker is then calculated by the software based on a preset coordinate system.

The drawback of such systems is that they only can be used in pre-designed labs, where the lighting is measured, cameras in their corresponding positions with respect to the human, and there should not be any shiny object in the field. Further, these systems require a direct line of sight between the subject performing a motion and three cameras to record the 3D position of markers. Also, these systems suffer from the movement artifact of the markers on skin.

Therefore, researchers cannot use this technique to record motion outside the lab. For example, if analyzing athletic motion, the participant should perform the motion in the lab in order for the scientist to extract the angles needed.

The 3D motion tracking exoskeleton can replace these techniques because:

- It does not require a line of sight.

- It does not suffer from the movement artifacts.

- Shiny objects will not affect the results.

- It can be used outside the lab.

\section{ACKNOWLEDGMENT}

First author would like to acknowledge the fund from King Saud University.

\section{References}

1. Bureau of Labor Statistics U.S Department of Labor, "Nonfatal occupational injuries and illnesses and requiring days away from work" http://www.bls.gov/news.release/archives/osh2_120 42009.pdf, 2008.

2. Alwasel, A. Elrayes, K. Abdel-Rahman, E. Haas, C., "Sensing Construction Work-related Musculoskeletal Disorders (WMSDs)", ISARC 2011, 2011.

3. Felscher, A. "Application note programmable angle sensor KMA200", 2004.

4. Alwasel, A. "A monitoring system to reduce shoulder injury among construction workers", MASc thesis, University of Waterloo, Waterloo, Canada, 2011.

5. Steering Committee for the Workshop on WorkRelated Musculoskeletal Injuries: The Research Base, National Research Council., "Work-Related Musculoskeletal Disorders Report and Workshop Summary", National Academy Press, Washington DC, 1999.

6. National Institute for Occupational Safety and Health., "Musculoskeletal disorders", 2011.

7. National Safety Council., "Management of WorkRelated Musculoskeletal Disorders", Accredited Standards Committee Z365, working draft, August 2002.
8. Bernard, B. Ed., "Musculoskeletal Disorders and Workplace Factors. A Critical Review of Epidemiologic Evidence for Work-Related Musculoskeletal Disorders of the Neck, Upper Extremity, and Low Back", National Institute for Occupational Safety and Health (NIOSH), 1997.

9 Svendsen, S. Bonde, J. Mathiassen, StengaardPedersen, S. Frich, L. "Work related shoulder disorders: quantitative exposure-response relations with reference to arm posture", Occupational and Environmental Medicine, vol. 61, pp. 844 - 853, OCT, 2004.

10 Roetenberg, D. Luinge, H. Slycke, P., "Xsens MVN: Full 6DOF Human Motion Tracking Using Miniature Inertial Sensors", Xsense Techologies, 2009.

11 Xsense 3D motion tracking systems, "MVN Biomech", http://www.xsens.com/en/mvn-biomech .

12 Roetenberg, D., Luinge, H.J., Baten, C.T., Veltink, P.H., "Compensation of Magnetic Disturbances Improves Inertial and Magnetic Sensing of Human Body Segment Orientation", IEEE Transactions on Neural Systems and Rehabilitation Engineering, vol. 13, no. 3, September 2005.

13 Brigante, M.N., Abbate, N., Basile, A., "Towards Miniaturization of a MEMS-Based Wearable Motion Capture System", IEEE Transactions on Industrial Electronics, vol. 58, no. 8, August 2011. 